SELECTED PAPER AT NCSP'19

\title{
Theoretical Analysis of Frame Error Detecting Schemes for Optical-Wireless Advanced Framed-DOOK System
}

\author{
Yuta Asano, Ran Sun, Hiromasa Habuchi and Yusuke Kozawa \\ Graduate School of Science and Engineering, Ibaraki University \\ 4-12-1 Nakanarusawa, Hitachi, Ibaraki 316-8511, Japan \\ E-mail: 17nd302t@vc.ibaraki.ac.jp \\ hiromasa.habuchi.hiro@vc.ibaraki.ac.jp \\ yusuke.kozawa.phd@vc.ibaraki.ac.jp
}

\begin{abstract}
In optical-wireless communications, an advanced frameddifferential on-off keying (AF-DOOK) system has been proposed by the authors. The AF-DOOK system can solve an optimum decision level adjustment problem and bit-slip problem in the conventional on-off keying (OOK) system. Moreover, the AF-DOOK system can detect frame errors by frame error detecting schemes, named the parity check method and the polarity check method. In this study, the polarity check method for the AF-DOOK system is investigated. In particular, the performance of frame error detection is evaluated by theoretical analysis. As a result, for the AF-DOOK system, the frame error detection probability of the polarity check method is found to be better than that of the parity check method. Moreover, it is clarified that there is a minimum frame error misdetection probability.
\end{abstract}

\section{Introduction}

In optical wireless communications using intensity modulation/direct detection schemes, to avoid nonlinear characteristics of the optical transmitter and photodiode, binary modulation schemes are studied [1]-[6]. On-off keying (OOK) is one of the well-known binary modulation schemes. OOK is a modulation that expresses information data by pulses. Therefore, the transmission efficiency achieves a valve of 1 [bit/slot], where the slot width equals the pulse duration. In OOK, to solve an optimum decision level adjustment problem and bit-slip problem, the framed-differential OOK (framedDOOK) system with a 1-bit synchronization signal has been proposed [7]. To improve the synchronization performance of the framed-DOOK system, we proposed the advanced framed-DOOK (AF-DOOK) system [8]. In the AF-DOOK system, the synchronization signal consists of two bits. The AF-DOOK system can detect a frame error using the first bit of an embedded 2-bit synchronization signal as a parity bit [9]. Moreover, since positive pulses alternate with negative ones on the output signal of the differential detector, the AFDOOK system has another criterion for the detection of frame errors, that is, the polarity check method.

In this paper, we show frame error detecting schemes of the AF-DOOK system: the parity check method and the polarity check method. We derive theoretical formulas for the frame error detecting performance of the polarity check method: frame error detection probability, frame error undetection probability, and frame error misdetection probability. Moreover, we compare the performance of the polarity check method with that of the parity check method by theoretical analysis.

\section{AF-DOOK System}

Figure 1 shows a block diagram of the AF-DOOK system and Fig. 2 shows the signal flow of the AF-DOOK system.

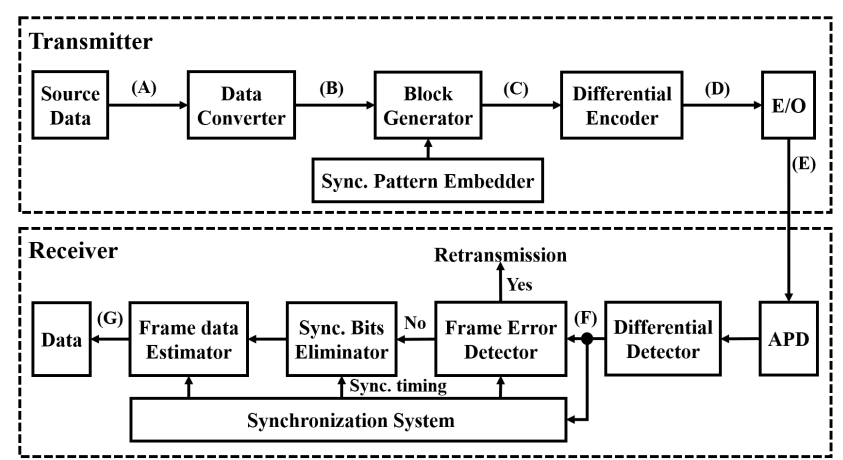

Figure 1: Block diagram of the AF-DOOK system

As shown in Fig. 1, in the AF-DOOK system, (A) source data is divided into frames every $M$ bits, and (B) the synchronization patterns $\left\{p_{1}, p_{2}, \cdots, p_{n}\right\}$ are embedded in every frame. (C) The bit pattern $\left\{s_{i 1} s_{i 2}\right\}$ for embedding the synchronization signal in each frame is 01 or 11 , and it is decided according to the following rules.

Rule (1): the case of $p_{i}=p_{i-1}$

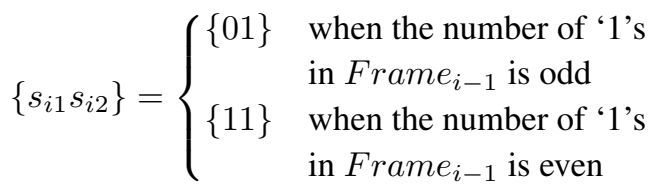




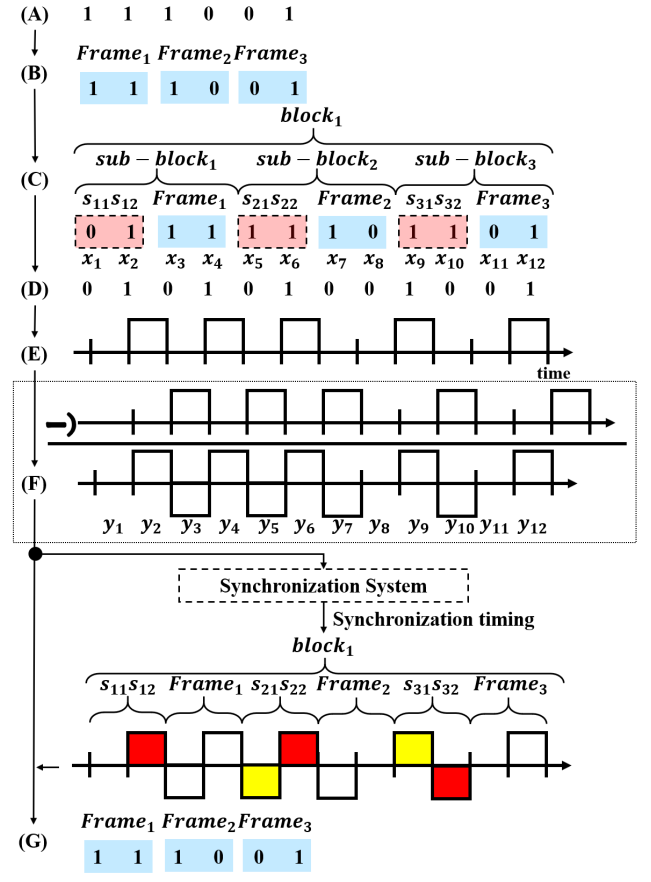

Figure 2: Signal flow of the AF-DOOK system

Rule (2): the case of $p_{i} \neq p_{i-1}$

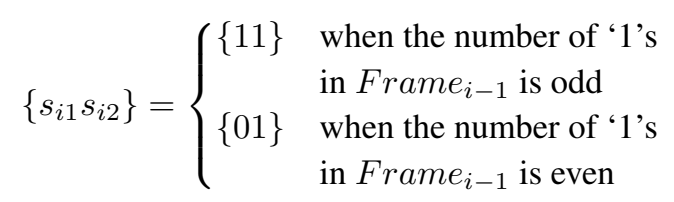

After embedding the synchronization signal, (D) this signal stream is differentially encoded and (E) transmitted as an optical signal. (F) The receiver detects the received signal by the differential detector and acquires synchronization timing by correlating the differential detected signal with the referential signal. (G) After acquiring the synchronization timing, the receiver eliminates the synchronization signal and estimates the message data.

\section{Frame Error Detecting Schemes}

The AF-DOOK system can detect a frame error by two frame error detecting schemes: the parity check method [9] and polarity check method. Figure 3 illustrates four examples of frame error detection by the polarity check method. Figure 3(I) shows a frame with no signal error. When there is no error in the signal, a positive polarity and a negative polarity appear alternately. In Fig. 3(II), the output of the differential detector has the same polarity. Therefore, the polarity check method can detect a frame error. When the number of errors in a frame is even, the parity check method invariably misses a frame error. However, the polarity check method can detect the frame error in some cases. In Fig. 3(III), the positive signals and negative signals appear alternately, although some

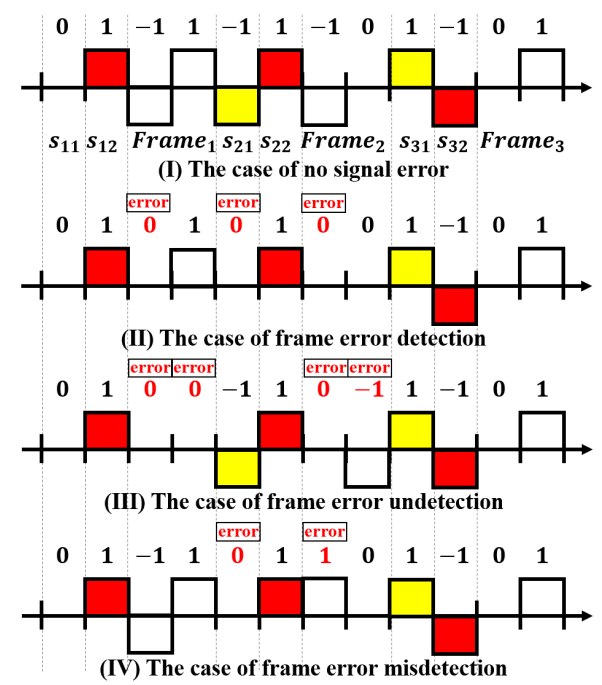

Figure 3: Examples of frame error detection

pulses become ' 0 '. Therefore, the polarity check method cannot detect the frame error. In Fig. 3(IV), there is only a parity bit error or some polarity errors in a frame. Here, the polarity error means that a positive signal is mistaken for a negative one or vice versa. Although symbol errors exist in a frame, message data may be correct. Then, the polarity check method may detect this frame as an error frame.

\section{Theoretical Analysis}

In this section, we evaluate the performances of the frame error detection methods by theoretical analysis. The performances of frame error detection are expressed by the frame error detection probability, $P_{\text {det }}$, the frame error undetection probability, $P_{\text {undet }}$, and the frame error misdetection probability, $P_{\text {miss }}$. The relationship among these probabilities is shown in Fig. 4.

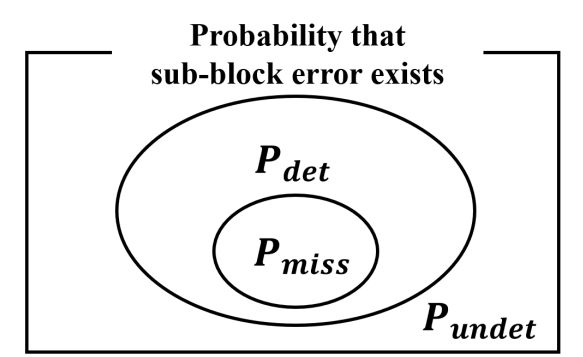

Figure 4: Relation among the probabilities

Moreover, we compare the polarity check method with the parity check method.

\subsection{Theoretical formulas}

There are the following undetected patterns in frame error 
detection of polarity check method.

$$
\begin{aligned}
& \text { Pattern 1: }\left(x_{j}, x_{j+1}\right)=(0,0) \rightarrow\left(y_{j}, y_{j+1}\right)=( \pm 1, \mp 1) \\
& \text { Pattern 2: }\left(x_{j}, x_{j+1}\right)=(1,1) \rightarrow\left(y_{j}, y_{j+1}\right)=(0,0) \\
& \text { Pattern 3: }\left(x_{j}, x_{j+1}\right)=(0,1) \rightarrow\left(y_{j}, y_{j+1}\right)=( \pm 1,0) \\
& \text { Pattern 4: }\left(x_{j}, x_{j+1}\right)=(1,0) \rightarrow\left(y_{j}, y_{j+1}\right)=(0, \pm 1)
\end{aligned}
$$

When the occurrence probabilities of ' 0 ' and ' 1 ' on the transmitter are equal, the occurrence probability of ' 0 ' is 0.5 and the occurrence probability of ' \pm 1 ' is 0.5 in the output of the differential detector. Therefore, the probability of occurrence of undetected patterns $P_{u n}$ is given by

$$
\begin{aligned}
P_{u n} & =\frac{1}{4} P( \pm 1 \mid 0) P(\mp 1 \mid 0)+\frac{1}{4} P(0 \mid 1) P(0 \mid 1) \\
& +\frac{1}{4} P( \pm 1 \mid 0) P(0 \mid 1)+\frac{1}{4} P(0 \mid 1) P( \pm 1 \mid 0)
\end{aligned}
$$

where $P(y \mid x)$ is the conditional probability. In the AFDOOK system, the sub-block consists of $M$ information bits and 2-bit synchronization signals. Since we consider that $M+1$ points exist at the bit position that an error is produced, other than the second bit in the synchronization signal, $P_{\text {undet }}$ is given by

$$
P_{\text {undet }}=\sum_{k=1}^{\lfloor(M+1) / 2\rfloor} \sum_{\ell=0}^{M+1-2 k} \frac{\left(\begin{array}{c}
M+1-k-\ell \\
\ell
\end{array}\right) P_{u n}^{k}\left(\frac{1}{2}\right)^{\ell}\left(1-P_{e S}\right)^{M+1-2 k-\ell}}{1-\left(1-P_{e S}\right)^{M+1}}
$$

where $k$ is the number of undetected patterns and $\ell$ is the number of occurrences of ' 0 's between undetected patterns. In Eq. (2), $(1 / 2)^{\ell}$ means the occurrence probability of ' 0 '. $P_{e S}$ expresses the symbol error rate (SER) of the AF-DOOK system. $P_{e S}$ is given by

$$
\begin{aligned}
P_{e S} & =\frac{1}{4}\left\{\operatorname{erfc}\left(\frac{T_{2}-\mu_{10}}{\sqrt{2 \sigma_{10}^{2}}}\right)-\operatorname{erfc}\left(\frac{T_{1}-\mu_{10}}{\sqrt{2 \sigma_{10}^{2}}}\right)\right. \\
& +\frac{1}{2} \operatorname{erfc}\left(-\frac{T_{2}-\mu_{11}}{\sqrt{2 \sigma_{11}^{2}}}\right)+\frac{1}{2} \operatorname{erfc}\left(\frac{T_{1}-\mu_{11}}{\sqrt{2 \sigma_{11}^{2}}}\right) \\
& +\frac{1}{2} \operatorname{erfc}\left(-\frac{T_{2}-\mu_{00}}{\sqrt{2 \sigma_{00}^{2}}}\right)+\frac{1}{2} \operatorname{erfc}\left(\frac{T_{1}-\mu_{00}}{\sqrt{2 \sigma_{00}^{2}}}\right) \\
& \left.+\frac{1}{2} \operatorname{erfc}\left(-\frac{T_{2}-\mu_{10}}{\sqrt{2 \sigma_{10}^{2}}}\right)+\frac{1}{2} \operatorname{erfc}\left(\frac{T_{1}-\mu_{01}}{\sqrt{2 \sigma_{01}^{2}}}\right)\right\}
\end{aligned}
$$

where $T_{1}$ and $T_{2}$ are threshold levels for the signal estimation of DOOK, $\mu_{10}, \mu_{01}, \mu_{00}$, and $\mu_{11}$ are the average of outputs of the differential detector [7], and $\sigma_{10}, \sigma_{01}, \sigma_{00}$ and $\sigma_{11}$ are the variances of the outputs of the differential detector [7]. Also, $P_{m i s s}$ is given by

$$
\begin{aligned}
P_{\text {miss }} & =P_{1}+P_{2} \\
& =\frac{\left(1-P_{e S}\right)^{M} P_{e S}+\sum_{m=1}^{M}\left(\begin{array}{c}
M \\
m
\end{array}\right) P( \pm 1 \mid \mp 1)^{m}\left(1-P_{e S}\right)^{M-m}}{1-\left(1-P_{e S}\right)^{M+1}}
\end{aligned}
$$

where $m$ is the number of occurrences of the polarity error. $P_{1}$ is the probability that only a parity symbol has a symbol estimation error. $P_{2}$ means the probability that there is a polarity error in a frame. From Fig. $4, P_{\text {det }}$ is given by

$$
P_{\text {det }}=1-P_{\text {undet }}-P_{\text {miss }}
$$

\subsection{Performance evaluation}

In this study, the performance of frame error detection by the polarity check method is compared with that of the parity check method by theoretical analysis, where the numerical conditions are shown in Table 1 [10]. The optical-wireless channel model and theoretical formulas of the parity check method are taken from [9].

Table 1: Parameters [10]

\begin{tabular}{l|l}
\hline Name & Value \\
\hline $\begin{array}{l}\text { Synchronization pattern } \\
\text { Number of } \\
\text { information bits per block }\end{array}$ & $\{1,1,-1\}$ \\
\hline $\begin{array}{l}\text { Number of } \\
\text { synchronization patterns } n\end{array}$ & 3 \\
\hline Slot interval $T_{s}$ & $\frac{1}{156 \times 10^{6}} \times \frac{M}{M+2}[\mathrm{~s}]$ \\
\hline Optical wavelength & $830[\mathrm{~nm}]$ \\
\hline Quantum efficiency $\eta$ & 0.6 \\
\hline $\begin{array}{l}\text { Logarithm variance of } \\
\text { scintillation } \sigma_{s}^{2}\end{array}$ & 0.01 \\
\hline APD gain & 100 \\
\hline Bulk leakage current $I_{b}$ & $1.09 \times 10^{-10}[\mathrm{~A}]$ \\
\hline Surface leakage current $I_{s}$ & $1.09 \times 10^{-8}[\mathrm{~A}]$ \\
\hline Boltzmann constant $K_{B}$ & $1.38065039 \times 10^{-23}$ \\
\hline Thermal noise $T_{r}$ & $300.0[\mathrm{~K}]$ \\
\hline Load resistor $R_{L}$ & $1030.0[\Omega]$ \\
\hline Modulation extinction ratio $M_{e}$ & $1.0[\%]$ \\
\hline
\end{tabular}

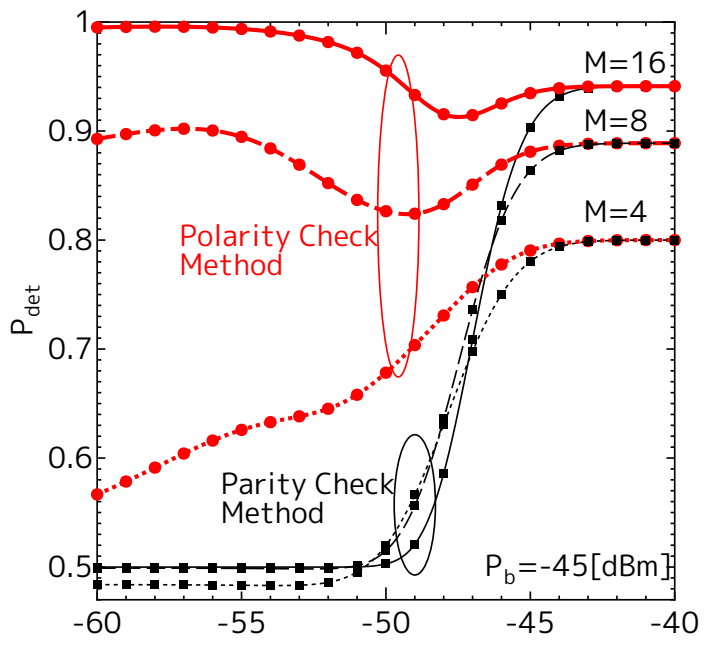

Average Transmission Power per bit, $\mathrm{P}_{\mathrm{t}}[\mathrm{dBm}]$

Figure 5: $P_{\text {det }}$ in the polarity check method and the parity check method

Figure 5 shows $P_{d e t}$ in the polarity check method and that of the parity check method. When $P_{t}$ is small, $P_{d e t}$ in the polarity check method is better than that in the parity check method. This is because the polarity check method can detect 
some frame errors whereas the parity check method invariably misses the frame error when the number of errors in a frame is even. However, since SER of the AF-DOOK system becomes similar to BER of the AF-DOOK system with increasing $P_{t}, P_{\text {det }}$ in the polarity check method shows the same performance as that in the parity check method with increasing $P_{t}$. Moreover, since the number of patterns that the parity check method cannot detect decreases with increasing $P_{t}$, a $P_{t}$ value at which $P_{\text {det }}$ in the polarity check method becomes minimum exists.

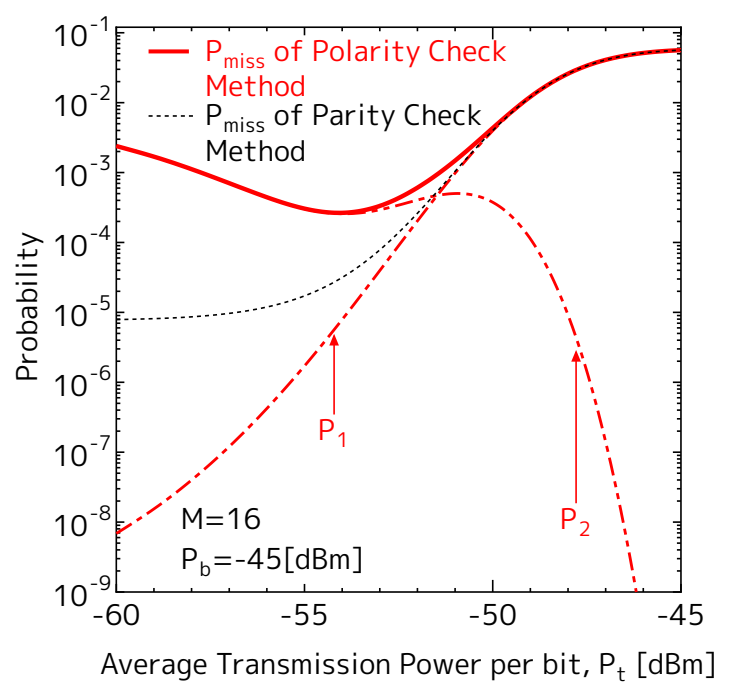

Figure 6: $P_{\text {miss }}$ in the polarity check method and the parity check method

Figure 6 shows $P_{m i s s}$ in the polarity check method and that in the parity check method when $M=16$. When $P_{t}$ is small, $P_{m i s s}$ in the parity check method is better than that in the polarity check method. That is because not only the patterns in which a parity error exists in a sub-block but also the patterns in which some polarity errors exist in a frame are misdetected by the polarity check method. However, since the probability of a polarity error becomes small with increasing $P_{t}, P_{\text {miss }}$ in the polarity check method becomes similar to that in the parity check method. Moreover, $P_{m i s s}$ in the polarity check method has a minimum. From Eq. (4), $P_{\text {miss }}$ in the polarity check method is expressed by two components: $P_{1}$ and $P_{2}$. With increasing $P_{t}, P_{1}$ increases monotonically, but $P_{2}$ becomes small. Thus, in the polarity check method, it is found that there is a minimum $P_{\text {miss }}$.

When $P_{t}$ is small, since $P_{m i s s}$ in the polarity check method is larger than that in the parity check method, the number of redundant retransmissions in the polarity check method is more than that by the polarity check method. However, $P_{d e t}$ in the polarity check method is considerably higher than that in the parity check method. Therefore, we are convinced that the performance of frame error detection by the polarity check method is better than that by the parity check method.

\section{Conclusion}

We presented frame error detection schemes for the AFDOOK system: the polarity check method and the parity check method. In particular, we investigated the frame error detection probability and the frame error misdetection probability by theoretical analysis. As the result, for the AF-DOOK system, we showed that the performance of frame error detection by the polarity check method is better than that by the parity check method.

\section{Acknowledgement}

This study was supported in part by a Grant-in-Aid for Scientific Research (C).

\section{References}

[1] Y. Tanaka, T. Komine, S. Haruyama and M. Nakagawa: Indoor visible light data transmission system utilizing white LED lights, IEICE Transactions on Communications, Vol. E86-B, No.8, pp. 2440-2454, 2003.

[2] J. M. Kahn and J. R. Barry: Wireless infrared communications, Proceedings of the IEEE, Vol. 89, No.2, pp. 265-298, 1997.

[3] H. Sugiyama, S. Haruyama and M. Nakagawa: Experimental investigation of modulation method for visiblelight communications, IEICE Transactions on Communications, Vol. E89-B, No. 12, pp. 3393-3400, 2006.

[4] D. Karunatilaka, F. Zafar, V. Kalavally and R. Parthiban: LED based indoor visible light communications: State of the art, IEEE Communication Surveys \& Tutorials., Vol. 17, No.3, pp. 1649-1678, 2015.

[5] H. Elgala, R. Mesleh and H. Haas: Indoor optical wireless communication: potential and state-of-the-art, IEEE Communication Magazine, Vol. 49, No.9, pp. 5662, 2011.

[6] H. Chan, K. L. Sterckx, J. M. H. Elmirghani and R. A. Cryan: Performance of optical wireless OOK and PPM systems under the constraints of ambient noise and multipath dispersion, IEEE Communications Magazine, Vol. 36, Issue. 12, pp. 83-87, 1998.

[7] Y. Matsuda, H. Habuchi and Y. Kozawa: Impact of improved on-off-keyed turbo-code system in optical wireless channel, RISP International Workshop on Nonlinear Circuits, Communications and Signal Processing 2016 (NCSP), 2016.

[8] Y. Asano, H. Habuchi and Y. Kozawa: Improved synchronization scheme for indoor visible-Light differential on-off keying, 2017 IEEE 6th Global Conference on Consumer Electronics (GCCE), pp. 394-398, 2017.

[9] Y. Asano, H. Habuchi and Y. Kozawa: Frame error detection performance of optical-wireless advanced framed-DOOK system, 12th International Conference on Signal Processing and Communication Systems (ICSPCS), 2018.

[10] M. Biagi, T. Borogovac and T. D. C. Little: Adaptive receiver for indoor visible light communications, Journal of Lightwave Technology, Vol. 31, No.23, pp.36763686, 2013. 